\title{
Factors influencing the long-term prognosis of patients with temporal lobe epilepsy: a single center study
}

\author{
Lu Yang ${ }^{1}$, Rui Zhang ${ }^{1}$, Haitao Zhu ${ }^{1}$, Fangqing Chen $^{1}$, Nian Yu ${ }^{2}$, Qing Di $^{2}$ \\ ${ }^{1}$ Epilepsy Center, The Affiliated Brain Hospital of Nanjing Medical University, Nanjing, China; ${ }^{2}$ Department of Neurology, The Affiliated Brain \\ Hospital of Nanjing Medical University, Nanjing, China \\ Contributions: (I) Conception and design: Q Di, L Yang; (II) Administrative support: R Zhang; (III) Provision of study materials or patients: R Zhang, \\ H Zhu, F Chen; (IV) Collection and assembly of data: L Yang; (V) Data analysis and interpretation: L Yang; (VI) Manuscript writing: All authors; (VII) \\ Final approval of manuscript: All authors. \\ Correspondence to: Qing Di. Department of Neurology, The Affiliated Brain Hospital of Nanjing Medical University, Nanjing, China. \\ Email: diqing1629@163.com.
}

Background: This study aimed to explore the risk factors influencing the long-term prognosis of temporal lobe epilepsy (TLE) patients treated with drug therapy or surgical treatment.

Methods: A total of 112 patients who were diagnosed with TLE were retrospectively enrolled. All of the patients were initially treated with antiepileptic drugs (AEDs). Patients who met the criteria of drugresistant TLE were evaluated for treatment with temporal lobectomy. The main outcome was whether the patients achieved seizure-free status. This was defined as when a patient experienced no seizures for at least 24 months of follow-up. The independent predictors of the outcome were evaluated using a multiple logistic regression model.

Results: The median follow-up period was 5 years (range, 2-13 years). At the end of follow-up, 26 patients had not achieved seizure freedom after treatment with AEDs, including 22 cases of monotherapy, 4 cases of duotherapy ( 1 case of drug-resistant epilepsy after continuous drug treatment). AED treatment was withdrawn in 6 patients. Overall, 23.2\% TLE patients (26/112) achieved seizure freedom after treatment with AEDs alone, while the remaining $76.8 \%$ (86/112) of patients were diagnosed as drug-resistant epilepsy (DRE). Of the 45 DRE patients who subsequently received surgical treatment, $77.8 \%(35 / 45)$ achieved seizure freedom, with successful AED withdrawal in 11 patients. The proportion of patients who achieved seizure-free status was higher among those who underwent temporal lobectomy than among those who continued with AED treatment (77.8\% vs. $23.2 \%, \mathrm{P}<0.001)$. Effective early monotherapy was an independent predictor for good therapeutic effect in all TLE patients (OR: 0.16; 95\% CI: 0.04-0.66; $\mathrm{P}=0.007)$. Multivariate logistic regression analysis showed the predictors of good prognosis in DRE patients after surgery to be unilateral origin of discharges in electroencephalogram (EEG, OR $=0.20,95 \% \mathrm{CI}$ : 0.06-0.74, $\mathrm{P}=0.016$ ), no secondary generalized tonic-clonic seizures (GTCS, OR $=0.08,95 \%$ CI: 0.01-0.67, $\mathrm{P}=0.002$ ), and not needing a subdural electrode ( $\mathrm{OR}=15.4,95 \% \mathrm{CI}: 1.36-174.38, \mathrm{P}=0.027$ ).

Conclusions: Effective early monotherapy was an independent protective factor for the favorable prognosis of TLE. Unilateral origin of discharges in EEG, no secondary GTCS, and not needing a subdural electrode were independent factors associated with favorable prognosis after TLE surgery.

Keywords: Temporal lobe epilepsy (TLE); epilepsy surgery; drug-resistant epilepsy; prognosis; risk factors

Submitted May 28, 2020. Accepted for publication Sep 02, 2020.

doi: 10.21037/apm-20-1415

View this article at: http://dx.doi.org/10.21037/apm-20-1415 


\section{Introduction}

Epilepsy, a common chronic brain disorder, is responsible for $1 \%$ of the global disease burden (1). Temporal lobe epilepsy (TLE) accounts for $30-35 \%$ of all epilepsies. The therapeutic response among TLE patients is heterogeneous. Previous studies have shown that while $5-42 \%$ of TLE cases respond well to routine antiepileptic drugs (AEDs) (2-4), as many as $50-70 \%$ of TLE cases are reported as drug-resistant epilepsy (DRE) (5-8). Surgery is recommended as an effective alternative treatment approach for drug-resistant TLE, especially for mesial TLE (9). It has been documented that after surgery, $60-70 \%$ of drugresistant TLE cases could achieve 1-2 years of seizure freedom (10). Unfortunately, at 5-10 years after surgery, the proportion of patients with seizure-free status is reportedly decreased to $30-40 \%$ (11), leading to some controversy surrounding which treatment is the most effective for TLE.

"Benign" TLE was recently reported in a clinical study, which found that some patients with TLE could maintain seizure-free status for more than 2 years even with hippocampal sclerosis (12). Therefore, it is necessary to reevaluate the long-term prognosis of TLE treatments in a larger sample size or different ethnic populations. Wiebe et al. conducted only one randomized controlled trial to explore the prognosis of TLE treated with either AED therapy or surgical therapy alone (13). Therefore, it is still necessary to establish which TLE cases would be most likely to benefit from treatment with AEDs. In addition, factors that will allow early identification of TLE patients who will respond well to surgery are also urgently needed. Thus, we performed a retrospective study to compare the prognoses of TLE patients treated with surgery or with AEDs alone, as well as the factors that influenced their outcomes, which will provide a foundation for more standardized clinical treatment of TLE. We present the following article in accordance with the STROBE reporting checklist (available at http://dx.doi.org/10.21037/apm-20-1415).

\section{Methods}

\section{Participants}

Patients who were diagnosed with TLE (14) between January 2006 and June 2017 in the Epilepsy Center of Nanjing Brain Hospital Affiliated to Nanjing Medical University. Patient information, including medical history and the results of nervous system examination at admission, video electroencephalogram (EEG), and brain magnetic resonance imaging (MRI), were collected.

A total of 95 patients who met the following diagnostic criteria were enrolled:

(I) Seizures were the exclusive symptom of TLE, and were usually accompanied by upset in the upper abdomen, autonomic dysfunction, and/or psychiatric disorders. Other supporting symptoms included sudden behavior arrest, gradual loss of consciousness, and limbic or oral automatisms of the mouth, with or without bilateral tonic-clonic seizures.

(II) EEG monitoring showed interictal or ictal epileptiform discharges from the temporal or frontotemporal region.

(III) Brain MRI presented structural abnormality of the temporal lobe or found no obvious abnormality.

All procedures performed in this study involving human participants were in accordance with the Declaration of Helsinki (as revised in 2013). This study was approved by the Ethics Committee of The Affiliated Brain Hospital of Nanjing Medical University (No. 2020-ky141-01).

\section{Treatment}

\section{Drug treatment}

Initially, the patients were treated with first-line AEDs for focal seizures according to the 2004 American Academy of Neurology/American Epilepsy Society (AAN/ AES) guidelines (15). First-line AEDs include valproic acid, carbamazepine, lamotrigine, oxcarbazepine and levetiracetam. The drug dosages were adjusted gradually.

\section{Surgical treatment}

Patients who satisfied the criteria for DRE underwent preoperative evaluation (16). Noninvasive evaluation methods, including video EEG, MRI, magnetoencephalogram, and positron emission tomography (PET), were used. If the epileptic foci could not be accurately located, a subdural cortical electrode was subsequently implanted to observe and determine the origin of the seizure onset. Signed informed consent was obtained from all patients who met the criteria for surgical resection after they had been provided with full information about epileptogenic focus resection surgery.

\section{Drug withdrawal}

Patients were advised to discontinue AED treatment if they achieved 1 year of seizure freedom after surgical treatment, as well as normal EEG findings. AED treatment should be withdrawn after 3 years. 


\section{Research methods}

Patient clinical data were obtained so that analysis of possible determinants of TLE prognosis could be performed. These included gender, age at seizure onset, duration of epilepsy, seizure frequency, febrile convulsion, history of brain injury, history of central nervous system infection, secondary generalized tonic-clonic seizures (GTCS), and drug treatment, as well as the findings of brain MRI and EEG, whether the patient had undergone subdural electrode implantation, and the pathological results of brain tissues. Duration of epilepsy was defined as the period from seizure onset to the end of follow-up for the patients who received AED therapy, or to the start of preoperative evaluation for patient who underwent surgery. Seizure frequency was defined as daily, weekly, or monthly. Drug treatment was defined as monotherapy and multitherapy. Interictal epileptiform discharges (IEDs) were defined as epileptiform discharges detected by EEG during the interictal interval period and were recorded as bilateral, unilateral, or normal. Ictal discharges (ID) were defined as a seizure origin pattern detected by EEG during the ictal interval period and were recorded as bilateral or unilateral. The findings of brain MRI were evaluated as negative (obvious abnormality) or positive (including hippocampal sclerosis, low-grade tumor, and cavernous hemangioma). The pathology results were based on the findings of resected brain tissues and were evaluated as positive (including hippocampal sclerosis, focal cortical dysplasia, cavernous hemangioma, tumor) or negative (no obvious abnormality or only gliosis).

\section{Follow-up and outcome}

Patients who received surgery during the observation period were followed up for at least 24 months after their operation. Patients who were not suitable for or who rejected surgery but continued the drug treatment were followed up for more than 24 months from the date of enrolment. The patients were followed up by telephone at an outpatient clinic every 3 months. Seizure freedom was defined as when a patient experienced no seizures for at least 24 months at the end of follow-up.

\section{Definition of benign TLE (17)}

Benign TLE was defined as when a patient achieved at least 2 years of seizure freedom during the observation period with or without the use of AEDs.

\section{Statistical analysis}

Quantitative data with approximately normal distribution were expressed as the mean $\pm \mathrm{SD}$. Quantitative data with skewed distribution were expressed as median percentiles. The means were compared by independent-samples $t$-tests. The proportions were compared by $\chi^{2}$ tests. The nonparametric tests were used for the enumeration data that were abnormally distributed. Univariate analysis was conducted, and the variables with $\mathrm{P} \leq 0.1$ were taken for multivariate binary logistic analysis. Hypothesis testing was performed by a bilateral test with the significance level set at $\mathrm{P}=0.05$. Statistical analyses were performed with SPSS 16.0 (IBM, New York, USA).

\section{Results}

\section{Basic clinical characteristics of all enrolled patients}

A total of 112 patients with TLE were included in this study. The follow-up time ranged from 2 years to 13 years, with a median of 5 years. By the end of follow-up, 45 cases (the surgery group) had received surgery, which was performed by the same neurosurgeon, and 67 cases (the AEDs group) had been treated with AEDs alone. Transient oculomotor nerve palsy was observed in three cases after surgery. No fatal postoperative complications were experienced.

There were no significant differences in age, gender, course of disease, seizure frequency, or history of febrile convulsion between the surgery group and the AEDs group. The basic clinical characteristics of the two groups are listed in Table 1.

\section{Prognosis of patients with TLE}

Of the 112 patients with TLE who were treated with AEDs, 26 patients became seizure free after treatment. Of these patients, 22 were treated with monotherapy and 2 were treated with multi-drug therapy (1 case developed DRE and then achieved seizure-free status after drug treatment). AEDs were successfully discontinued in 6 of the patients. Overall, $23.2 \%$ of the patients became seizure free after drug treatment. Of the 112 cases, 87 cases were diagnosed as DRE after drug treatment, including 3 cases who had bilateral TLE and were not suitable for surgical treatment. 
Table 1 Comparisons of basic clinical characteristics between surgery group and the AEDs group

\begin{tabular}{|c|c|c|c|}
\hline Variable & Surgery group $(n=45)$ & AEDs group $(n=67)$ & $P$ value \\
\hline M & $20(44 \%)$ & 35 (52\%) & \\
\hline $\mathrm{F}$ & $25(56 \%)$ & 32 (48\%) & \\
\hline Age at onset & $20.44 \pm 11.80$ & $24.10 \pm 13.83$ & 0.988 \\
\hline Seizure frequency & & & 0.183 \\
\hline Daily & $3(7 \%)$ & $2(2 \%)$ & \\
\hline Weekly & $22(49 \%)$ & $16(24 \%)$ & \\
\hline Monthly & $20(44 \%)$ & $49(72 \%)$ & \\
\hline No & $31(69 \%)$ & $44(66 \%)$ & \\
\hline CNS infection & & & 0.288 \\
\hline Yes & $5(11 \%)$ & $11(16 \%)$ & \\
\hline No & $40(89 \%)$ & $56(84 \%)$ & \\
\hline
\end{tabular}

AEDs, antiepileptic drugs; CNS infection, central nervous system infection.

Of the 87 DRE patients, 45 cases underwent surgery and 39 cases continued to maintain AED treatment because they or their relatives refused surgery. Of the patients treated with surgery, 35 became seizure free, as shown in Figure 1. The seizure-free proportion of surgery group was significantly higher than that of the AEDs group $(77.8 \%$ vs. $23.2 \%$, $\mathrm{P}<0.001)$. AEDs were successfully discontinued in 11 of the patients who underwent surgical treatment. Figure 2 shows the types of AEDs used to treat the 112 patients.

\section{Factors affecting the prognosis of TLE patients who receive drug treatment}

Univariate factor analysis showed that late seizure onset, short epilepsy duration, and a good response to early monotherapy were related to a favorable prognosis in TLE patients treated with AEDs. The factors affecting the prognosis of the TLE patients who received drug treatment are listed in Table 2. Multivariate logistic regression analysis revealed that effective early monotherapy $(\mathrm{OR}=0.21,95 \%$ CI: $0.05-0.89, \mathrm{P}=0.034)$ was an independent protective factor of good outcome in TLE patients after drug treatment.

\section{Factors affecting the prognosis of patients with drug-resistant TLE after surgical treatment}

Univariate analysis showed that no secondary GTCS and unilateral origin of discharges in EEG were associated with a favorable prognosis in drug-resistant TLE patients after surgery. The details of factors affecting the prognosis of patients with drug-resistant TLE after surgery are listed in Table 3. Multivariate logistic regression analysis revealed that unilateral origin of discharges in EEG (OR $=0.20,95 \%$ CI: $0.06-0.74, \mathrm{P}=0.016$ ), no secondary GTCS (OR $=0.08$, 95\% CI: $0.01-0.67, \mathrm{P}=0.002)$, and not needing a subdural electrode (OR $=15.4,95 \%$ CI: $1.36-174.38, \mathrm{P}=0.027)$ were independent predictors of a good prognosis in patients with drug-resistant TLE after surgery.

\section{Discussion}

With over $50 \%$ of focal epilepsies originating in the temporal lobe, TLE is one of the most common focal epilepsies. The incidence of DRE is widely understood to be higher in TLE than in other epilepsies. In the current study, 112 TLE patients were initially treated with AEDs, but only 26 of them $(23.2 \%)$ were seizure free for more 


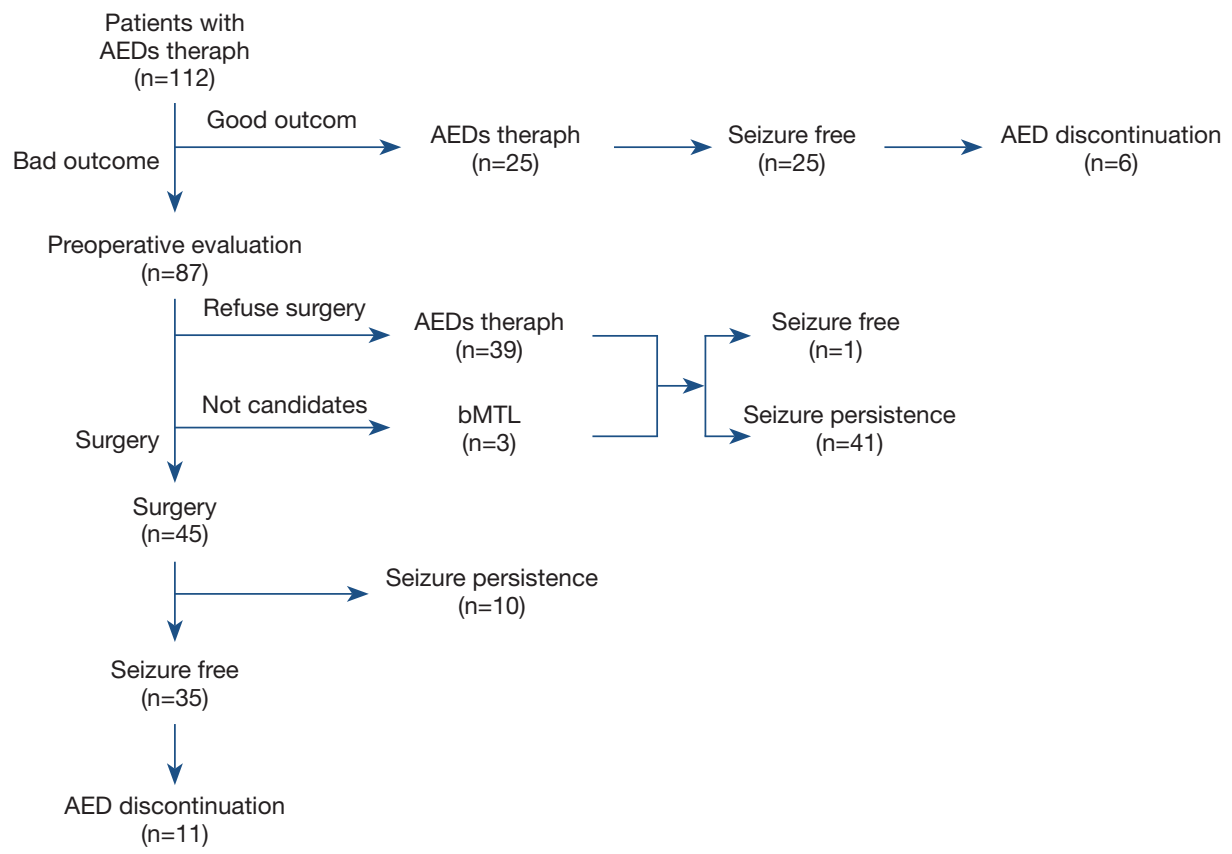

Figure 1 Longitudinal seizure outcomes. Good outcome: seizure free for at least 2 years; poor outcome: seizures continued. AEDs, antiepileptic drugs; BTLE, bilateral TLE.

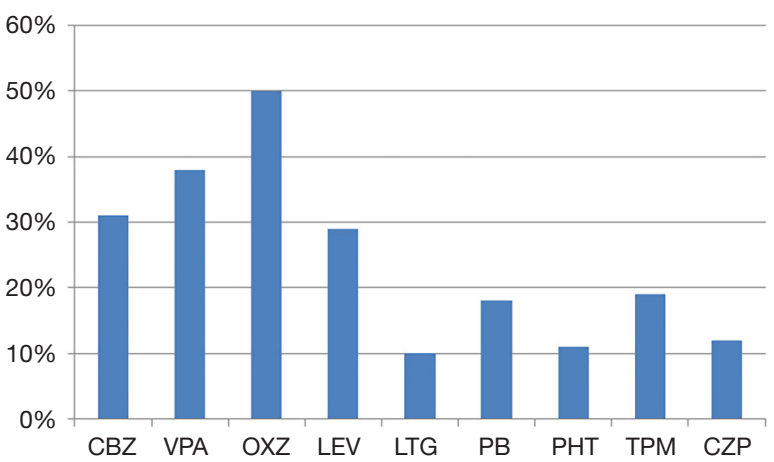

Figure 2 AEDs used to treat the patients with TLE. CBZ, carbamazepine; VPA, sodium valproate; OXZ, oxcarbazepine; LEV, lamotrigine; LEV, levetiracetam; PB, phenobarbital; PHT, phenytoin sodium; TPM, topiramate; CZP, clonazepam; AEDs, antiepileptic drugs; TLE, temporal lobe epilepsy.

than 24 months. Previous studies have reported 12-20\% to be a well-controlled seizure rate for adult TLE patients treated with AEDs $(18,19)$. Epidemiological studies also indicated that a small proportion of TLE cases experienced a spontaneous remission period whether AEDs were used or not (20,21). Hernández-Ronquillo et al. (22) found that
$29 \%$ of patients with TLE had a "mild course", implying that seizure-free status or good control could be naturally achieved without AEDs. The good control rate of seizures with AEDs among the TLE patients in our present study was significantly lower than the total seizure control rate (60-70\%). The lower control rate of seizures in this study may be attributable to a tendency of medically refractory epilepsy among our TLE patients, or our strict definition of a seizure-free period. In 2010, ILAE defined the seizurefree period during treatment as three times the longest interval between seizures before treatment or 1 year without seizures, with the longer period of the two being adopted. In the present study, the definition of benign TLE was used to define the seizure-free period as at least 2 years.

This study examined the clinical characteristics of benign TLE. It was discovered that effective monotherapy in the early stage was an independent protective factor for the favorable prognosis of TLE. This was consistent with the results of previous studies showing the relationship between the number of AEDs used and the therapeutic effect (16). This meant that the highest seizure-free rate was profoundly predicted by the effectiveness of the first AED; however, with the addition or adjustment of drugs used, the seizurefree rate decreased. Stefanatou et al. (23) also found that the 
Table 2 Predictors of prognosis in TLE patients who receive drug treatment

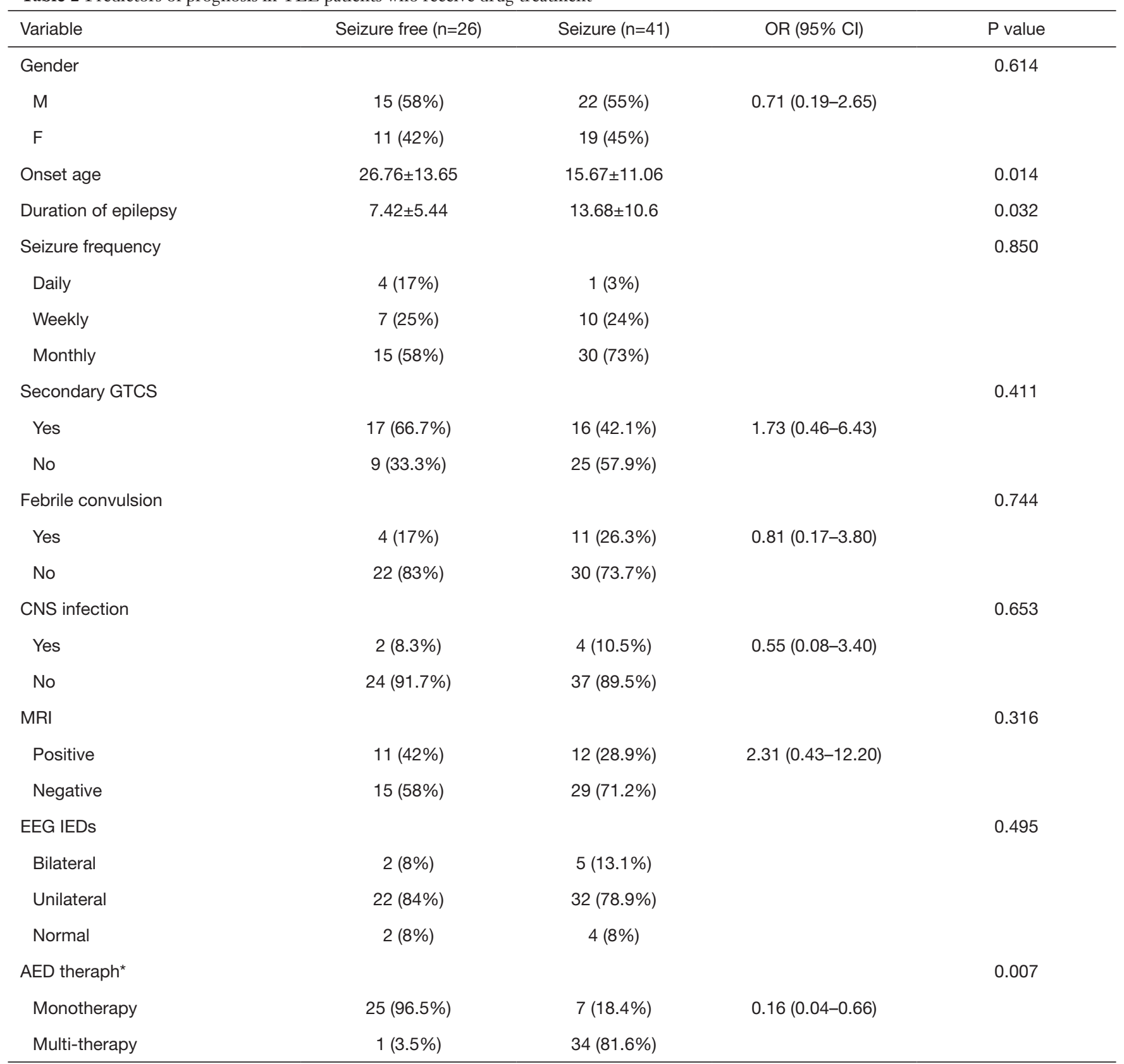

*, independent risk factors for relapse significantly at $\mathrm{P}<0.05$ after multivariate analysis. TLE, temporal lobe epilepsy; GTCS, generalized tonic-clonic seizures; CNS infection, central nervous system infection; MRI, magnetic resonance imaging; EEG, electroencephalogram; IEDs, interictal epileptiform discharges; ID, ictal discharges. 
Table 3 Predictors of prognosis in patients with drug-resistant TLE after surgery

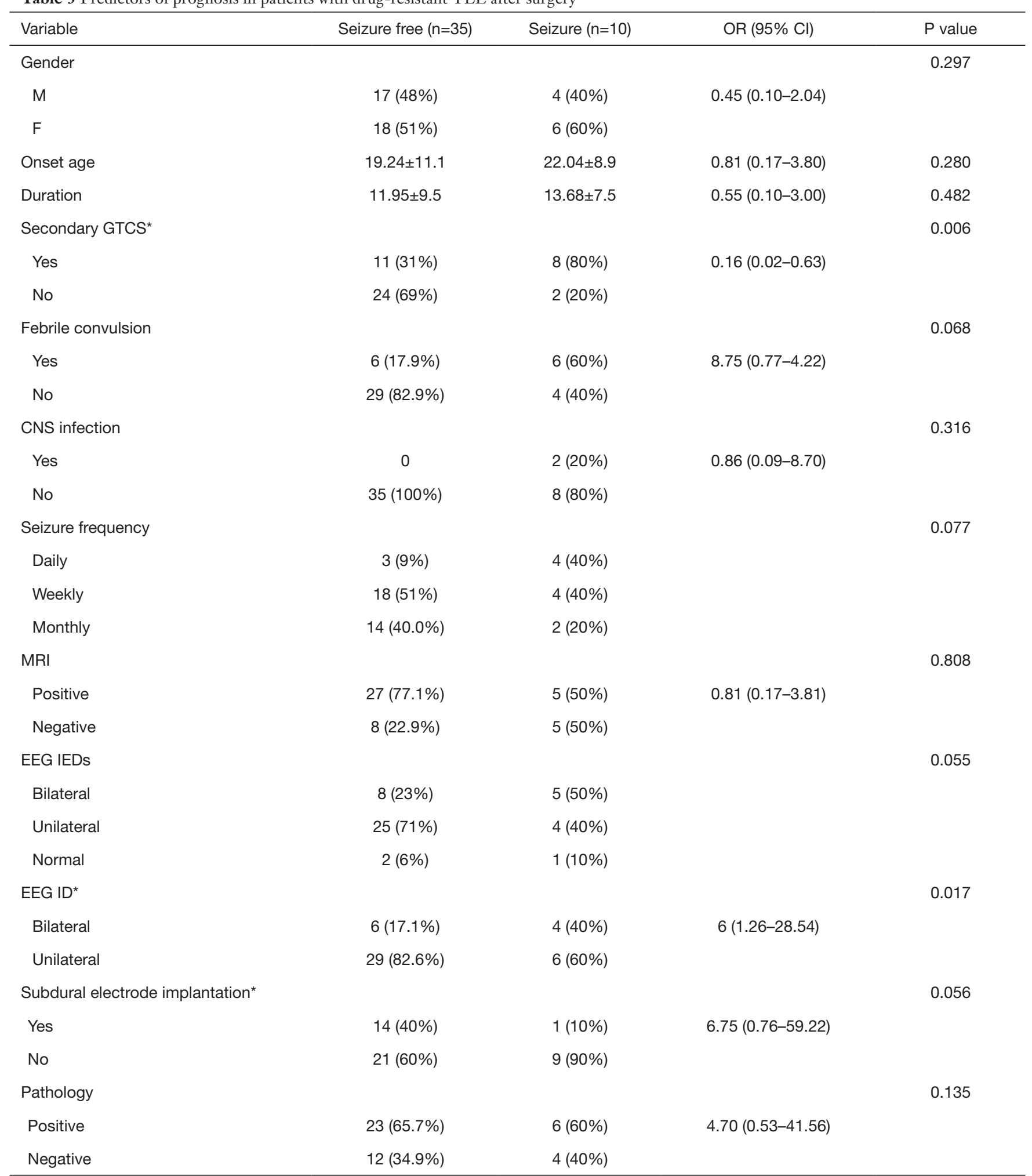

*, independent risk factors for relapse significantly at $\mathrm{P}<0.05$ after multivariate analysis. TLE, temporal lobe epilepsy; GTCS, generalized tonic-clonic seizures; CNS infection, central nervous system infection; MRI, magnetic resonance imaging; EEG, electroencephalogram; IEDs, interictal epileptiform discharges; ID, ictal discharges. 
number of drugs previously used was positively correlated with drug resistance. Univariate analysis in this study also showed that late onset and short disease duration were both correlated to a favorable prognosis of TLE treated with drug therapy. Moreover, Aguglia et al. (24) followed up 190 patients with non-lesion TLE who were treated with drugs for 10 years and found that later onset of epilepsy was correlated with a better prognosis. An older age at onset ( $>18$ years old) was identified as an independent predictor for a favorable prognosis after drug treatment. The authors described that the rate of recurrence in adults with epilepsy was lower than that in children; a poor prognosis of epilepsy was notably related to a younger age at onset (25-27).

The development of drug resistance in TLE is timedependent, which means that the probability of patients developing drug resistance increases as the disease course increases. Berg's (28) multi-center study followed up 333 patients with epilepsy. It was found that the incubation period from first onset to resistance to the second AED was 9.1 years. Approximately $26 \%$ of DRE patients had a remission period of more than 1 year before surgery, and as many as $8.5 \%$ of DRE patients had a remission period of more than 5 years. Therefore, it could be speculated that the rate of DRE may increase with the follow-up time. Thus, some patients with benign TLE may also be sensitive to drug treatment in the early stage.

It can be expected that some patients with benign TLE will also show the trend of drug resistance as their disease progresses. In this study, 86/112 (76.8\%) of TLE patients developed DRE after standardized drug treatment. TLE is a classic focal epilepsy, and a higher tendency of DRE than extra temporal lobe epilepsy was observed during long-term follow-up. Once epilepsy becomes drug resistant, surgical treatment is recommended. A long-term follow-up (median, 5 years) was performed on the patients with drug-resistant TLE who were treated with surgery in this study. At the end of the follow-up, $77.8 \%$ had seizure-free status for more than 2 years and 11 patients successfully discontinued drugs. Previous studies have reported that surgical treatment of TLE to be effective and the seizure-free rate after surgery to be around $62-80 \%(29,30)$.

The factors affecting the drug-resistance of TLE treated with surgery were complex and varied. This study found that not needing a subdural electrode, no secondary tonic-clonic seizures before surgery, and the unilateral origin of discharges in EEG during the ictal period were predictors of a favorable prognosis after surgery. The need for subdural electrode monitoring of epileptogenic lesions reflects the ease of preoperative evaluation. When the location and scope of the epilepsy focus are confirmed and the results from various non-invasive assessments are consistent, subdural electrode implantation is not required. Therefore, it is not difficult to understand why patients without subdural electrode implants had a good postoperative prognosis. On the contrary, patients with an electrode implanted were usually diagnosed as lateral TLE without obvious related lesions on brain MRI, and the postoperative prognosis was poorer than that of patients with medial TLE.

Preoperative tonic-clonic seizures were an independent risk factor for a poor prognosis. We speculated that frequent preoperative secondary tonic-clonic seizures could lead to comprehensive cerebral hypoxia and brain injury, which in turn can result in secondary potential epileptogenic lesions. On the other hand, classic medial TLE was less likely to result in secondary tonic-clonic seizures. A patient suffering more of these seizures before surgery may suggest the possibility of temporal plus epilepsy, which increases the difficulty of the preoperative evaluation as well as the extent of resection, and therefore affects the prognosis. McIntosh et al. (31) investigated the correlation between the preoperative factors and postoperative efficacy, and found that patients who had obvious lesions on imaging and experienced no secondary tonic-clonic seizures before surgery had a high incidence of becoming seizure-free after the operation. We also found that the unilateral origin of discharges in EEG during the ictal period was an independent protective factor that predicted a good prognosis after surgery. Tatum et al. (32) observed the rhythmic origin of EEG in the temporal area of 39 patients with medial TLE by PET/single photon emission computed tomography (SPECT) and the Wada test in the seizure period before surgery. They found that only the rhythmic origin of EEG in the temporal area during the ictal period was correlated with a favorable prognosis after surgery. Lau et al. (33) compared a group of patients with negative brain MRI findings with a group of patients with unilateral hippocampal sclerosis and discovered that the good control rate of postoperative epilepsy was related to the unilateral origin of discharges in EEG during the ictal period, but it was not associated with the presence or absence of hippocampal sclerosis. Brain MRI and EEG are considered as two significant laboratory examinations for the preoperative evaluation of DRE. This study did not find that the lesions detected by brain MRI were related to the prognosis of epilepsy after surgery. Some researchers (32) 
believe that EEG during the ictal period is an important tool for predicting the prognosis of postoperative epilepsy when the findings of brain MRI are normal.

\section{Conclusions}

TLE is an epilepsy syndrome that presents with high heterogeneity of therapeutic responses. It is therefore necessary to dynamically explore the long-term prognosis of TLE patients with drug therapy alone or surgical therapy on as well as the influencing factors. This study showed that $12.6 \%$ of TLE patients were defined as having benign TLE, with the patients achieving seizure-free status for at least 2 years with drug therapy. Effective early monotherapy to early monotherapy was an independent protective factor for the favorable prognosis of TLE. Of the patients with drug-resistant TLE who received surgery, $77.8 \%$ stayed seizure free for at least 2 years after surgery. The occurrence of secondary GTCS before surgery was an independent risk factor for a poor prognosis after surgery. Additionally, not needing a subdural electrode and the unilateral origin of discharges in EEG during the ictal period were also independent protective factors for favorable postoperative prognosis.

\section{Acknowledgments}

Funding: None.

\section{Footnote}

Reporting Checklist: The authors have completed the STROBE reporting checklist. Available at http://dx.doi. org/10.21037/apm-20-1415

Data Sharing Statement: Available at http://dx.doi. org/10.21037/apm-20-1415

Conflicts of Interest: All authors have completed the ICMJE uniform disclosure form (available at http://dx.doi. org/10.21037/apm-20-1415). The authors have no conflicts of interest to declare.

Ethical Statement: The authors are accountable for all aspects of the work in ensuring that questions related to the accuracy or integrity of any part of the work are appropriately investigated and resolved. All procedures performed in this study involving human participants were in accordance with the Declaration of Helsinki (as revised in 2013). This study was approved by the Ethics Committee of The Affiliated Brain Hospital of Nanjing Medical University (No. 2020-ky141-01). Signed informed consent was obtained from all patients.

Open Access Statement: This is an Open Access article distributed in accordance with the Creative Commons Attribution-NonCommercial-NoDerivs 4.0 International License (CC BY-NC-ND 4.0), which permits the noncommercial replication and distribution of the article with the strict proviso that no changes or edits are made and the original work is properly cited (including links to both the formal publication through the relevant DOI and the license). See: https://creativecommons.org/licenses/by-nc-nd/4.0/.

\section{References}

1. Guazzi M, Striano P. GABA strikes down again in epilepsy. Ann Transl Med 2019;7:57.

2. Bilevicius E, Yasuda CL, Silva MS, et al. Antiepileptic drug response in temporal lobe epilepsy: a clinical and MRI morphometry study. Neurology 2010;75:1695-710.

3. Gambardella A. Benign temporal lobe epilepsy. Epilepsia 2010;51:45-6.

4. Varoglu AO, Saygi S, Acemoglu H, et al. Prognosis of patients with mesial temporal lobe epilepsy due to hipocampal sclerosos. Epilepsy Res 2009;85:206-11.

5. Berg AT. Understanding the delay before epilepsy surgery: who develops intractable focal epilepsy and when? CNS Spectr 2004;9:136-44.

6. Berg AT. The natural history of mesial temporal lobe epilepsy. Curr Opin Neurol 2008;21:173-8.

7. Pittau F, Bisulli F, Mai R, et al. Prognostic factors in patients with mesial temporal lobe epilepsy. Epilepsia 2009;50:41-4.

8. Sànchez J, Centanaro M, Solís J, et al. Factors predicting the outcome following medical treatment of mesial temporal epilepsy with hippocampal sclerosis. Seizure 2014;23:448-53.

9. Stephen LJ, Kwan P, Brodie MJ. Does the cause of localisation-related epilepsy influence the response to antiepileptic drug treatment? Epilepsia 2001;42:357-62.

10. Uijl SG, Leijten FS, Arends JB, et al. Prognosis after temporal lobe epilepsy surgery: the value of combining predictors. Epilepsia 2008;49:1317-23

11. Janszky J, Janszky I, Schulz R, et al. Temporal lobe epilepsy with hippocampal sclerosis: predictors for long- 
term surgical outcome. Brain 2005;128:395-404.

12. Labate A, Ventura P, Gambardella A, et al. MRI evidence of mesial temporal sclerosis in sporadic "benign" temporal lobe epilepsy. Neurology 2006;66:562-5.

13. Wiebe S, Blume WT, Girvin JP, et al. Effectiveness and Efficiency of Surgery for Temporal Lobe Epilepsy Study Group. A randomized, controlled trial of surgery for temporal-lobe epilepsy. N Engl J Med 2001;345:311-8.

14. Proposal for revised classification of epilepsies and epileptic syndromes. Commission on Classification and Terminology of the International League Against Epilepsy. Epilepsia 1989;30:389-99.

15. French JA, Kanner AM, Bautista J, et al. Efficacy and Tolerability of the New Antiepileptic Drugs I: Treatment of New Onset Epilepsy: Report of the Therapeutics and Technology Assessment Subcommittee and Quality Standards Subcommittee of the American Academy of Neurology and the American Epilepsy Society. Neurology 2004;62:1252-60

16. Kwan P, Brodie MJ. Early identification of refractory epilepsy. N Engl J Med 2000;342:314-9.

17. Labate A, Gambardella A, Andermann E, et al. Benign mesial temporal lobe epilepsy. Nat Rev Neurol 2011;7:237-40

18. Helmstaedter C, Kurthen M, Lux S, et al. Chronic epilepsy and cognition: a longitudinal study in temporal lobe epilepsy. Ann Neurol 2003;54:425-32.

19. Semah F, Lamy C, Demeret S. Hippocampal sclerosis and other hippocampal abnormalities in the early identifification of candidates for epilepsy surgery. Arch Neurol 2002;59:1042-3.

20. Hauser WA. The natural history of temporal lobe epilepsy. In: Luders HO, etidor. Epilepsy surgery. Raven Press, New York, 1992:133-41.

21. King MA, Newton MR, Jackson GD, et al. Epileptology of the first-seizure presentation: a clinical, electroencephalographic, and magnetic resonance imaging study of 300 consecutive patients. Lancet 1998;352:1007-11.

22. Hernández-Ronquillo L, Buckley S, Ladino LD, et al. How many adults with temporal epilepsy have a mild course and do not require epilepsy surgery? Epileptic Disord 2016;18:137-47.

23. Stefanatou M, Gatzonis S, Peskostas A, et al. Drugresponsive versus drug-refractory mesial temporal lobe epilepsy: a single-center prospective outcome study. Postgrad Med 2019;131:479-85.

24. Aguglia U, Beghi E, Labate A, et al. Age at onset predicts good seizure outcome in sporadic non-lesional and mesial temporal sclerosis based temporal lobe epilepsy. J Neurol Neurosurg Psychiatry 2011;82:555-9.

25. Hirtz DG, Ellenberg JH, Nelson KB. The risk of recurrence of non-febrile seizures in children. Neurology 1984;34:637-41.

26. Beghi E, Tognoni G. Prognosis of epilepsy in newly referred patients: a multicenter prospective study. Collaborative group for the study of epilepsy. Epilepsia 1988;29:236-43.

27. Hart YM, Sander JWAS, Johnson AL, et al. National general practice study of epilepsy: recurrence after a fifirst seizure. Lancet 1990;336:1271-4.

28. Berg AT, Langfitt J, Shinnar S, et al. How long does it take for partial epilepsy to become intractable? Neurology 2003;60:186-90.

29. de Tisi J, Bell GS, Peacock JL, et al. The long term outcome of adult epilepsy surgery, patterns of seizure remission, and relapse: a cohort study. Lancet 2011;378:1388-95.

30. Edelvik A, Rydenhag B, Olsson I, et al. Long-term outcomes of epilepsy surgery in Sweden: a national prospective and longitudinal study. Neurology 2013;81:1244-51.

31. McIntosh AM, Kalnins RM, Mitchell LA, et al. Temporal lobectomy: long-term seizure outcome, late recurrence and risks for seizure recurrence. Brain 2004;127:2018-30.

32. Tatum WO, Benbadis SR, Hussain A, et al. Ictal EEG remains the prominent predictor of seizure-free outcome after temporal lobectomy in epileptic patients with normal brain MRI. Seizure 2008;17:631-6.

33. Lau T, Miller T, Klein T, et al. Temporal lobe surgery in medically refractory epilepsy: A comparison between populations based on MRI findings. Seizure 2014;23:20-4.

(English Language Editor: J. Reynolds)

Cite this article as: Yang L, Zhang R, Zhu H, Chen F, Yu N, Di Q. Factors influencing the long-term prognosis of patients with temporal lobe epilepsy: a single center study. Ann Palliat Med 2020;9(5):3194-3203. doi: 10.21037/apm-20-1415 